\title{
AIP ${ }_{\text {cesoumalt }}^{\text {chemical Physics }}$
}

\section{Phase diagram of hard snowman-shaped particles}

Matthew Dennison, Kristina Milinković, and Marjolein Dijkstra

Citation: J. Chem. Phys. 137, 044507 (2012); doi: 10.1063/1.4737621

View online: http://dx.doi.org/10.1063/1.4737621

View Table of Contents: http://jcp.aip.org/resource/1/JCPSA6/v137/i4

Published by the American Institute of Physics.

\section{Additional information on J. Chem. Phys.}

Journal Homepage: http://jcp.aip.org/

Journal Information: http://jcp.aip.org/about/about_the_journal

Top downloads: http://jcp.aip.org/features/most_downloaded

Information for Authors: http://jcp.aip.org/authors

\section{ADVERTISEMENT}

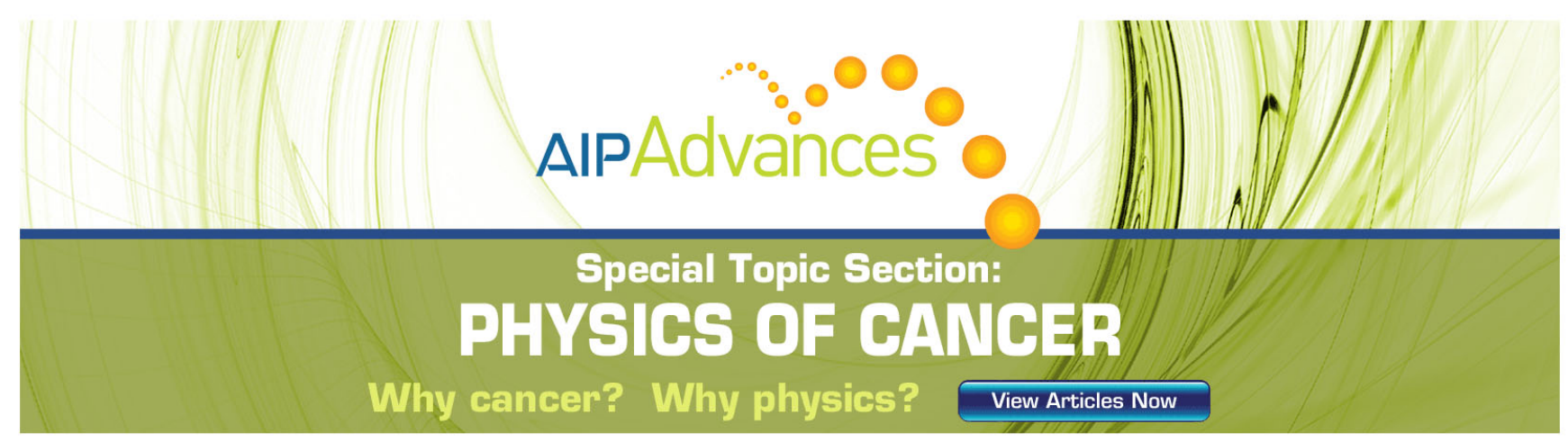




\title{
Phase diagram of hard snowman-shaped particles
}

\author{
Matthew Dennison, a) Kristina Milinković, and Marjolein Dijkstra \\ Soft Condensed Matter, Debye Institute for Nanomaterials Science, Utrecht University, Princetonplein 5 , \\ 3584 CC Utrecht, The Netherlands
}

(Received 5 April 2012; accepted 21 June 2012; published online 27 July 2012)

\begin{abstract}
We present the phase diagram of hard snowman-shaped particles calculated using Monte Carlo simulations and free energy calculations. The snowman particles consist of two hard spheres rigidly attached at their surfaces. We find a rich phase behavior with isotropic, plastic crystal, and aperiodic crystal phases. The crystalline phases found to be stable for a given sphere diameter ratio correspond mostly to the close packed structures predicted for equimolar binary hard-sphere mixtures of the same diameter ratio. However, our results also show several crystal-crystal phase transitions, with structures with a higher degree of degeneracy found to be stable at lower densities, while those with the best packing are found to be stable at higher densities. (C) 2012 American Institute of Physics. [http://dx.doi.org/10.1063/1.4737621]
\end{abstract}

\section{INTRODUCTION}

The potential for building colloidal structures with useful optical, mechanical and/or functional properties has lead to the development of new routes for synthesizing anisotropic colloids. ${ }^{1}$ Recent work has shown the prospect of controlling the size, shape and surface properties of colloidal particles, ${ }^{2,3}$ resulting in a huge number of possible building blocks. Understanding how the microscopic properties of these particles affect the macroscopic behavior of a system is therefore very important.

One such anisotropic colloidal particle with a simple asymmetric shape is the snowman particle, which consists of two spheres of different diameters joined together. Particles with this specific geometry can also be referred to as hetero-atomic dimers or asymmetric dumbbells. It is possible to synthesize snowman particles with a range of different diameter ratios of the two spheres and also different sphere separations, ${ }^{4-9}$ providing a large phase space even for purely repulsive interactions. These particles have the potential to mimic diatomic molecules and form a wide range of crystalline structures and it would clearly be desirable to understand their phase behavior. However, understanding the packing of colloidal particles with purely hard-body interactions has been a persistent challenge. Even for hard spheres, which are the simplest three-dimensional reference system and which have been studied since the early days of computer simulations, ${ }^{10,11}$ the issue of the relative stability of the face-centered-cubic (FCC) and hexagonal-close-packed (HCP) crystalline structures was a longstanding one. ${ }^{12-14}$ Particle anisotropy only adds to this complexity.

Perhaps the simplest model anisotropic particle is the hard dumbbell (or dimer), which can be seen as a special case of a snowman particle formed from hard spheres of equal diameter. Such systems have been extensively studied

a) Present address: Department of Physics and Astronomy, Vrije University, De Boelelaan 1081, 1081 HV Amsterdam, The Netherlands. in both two and three dimensions using theoretical ${ }^{15-17}$ and simulation ${ }^{18-22}$ approaches. The phase diagram of these particles is already surprisingly rich, ${ }^{22}$ with a variety of crystalline structures found to be stable. For the case of tangential spheres the system forms a stable crystalline phase where the constituent spheres sit on an FCC lattice and are randomly connected to form dimers. This so-called aperiodic FCC structure $^{19-22}$ offers an interesting parallel to the FCC structure of hard spheres. The similar nature of the crystalline phases of tangential dumbbell and hard-sphere systems suggests the intriguing prospect that other tangential dimer systems, such as snowman-shaped particles, can form binary hard-sphere crystalline phases, where the two spheres of the dimer would sit on the corresponding lattice sites of the binary crystal. Therefore, as well as being interesting anisotropic particles in their own right, snowman particles can also be viewed as a system of paired up spheres.

Binary hard-sphere mixtures are often used as a colloidal model of atomic systems. ${ }^{23-25}$ Several attempts have been made to determine the closest packed crystalline structures of binary hard sphere mixtures using theoretical ${ }^{26}$ and simulation methods. ${ }^{27-30}$ In particular, Ref. 29 found $\mathrm{NaCl}, \mathrm{CrB}$, $\alpha \mathrm{IrV}$ and $\gamma \mathrm{CuTi}$ structures to be close packed for various diameter ratios, and being able to form this rich variety of colloidal crystals would be desirable. However, studies of the phase behavior show that these crystal structures are often metastable with respect to other crystal structures or phase separated FCC structures. ${ }^{31}$ Fabrication of the best packed structures could be aided by using snowman-shaped particles, as phase separation would no longer be an issue since each large sphere is already tangential to a smaller sphere. Stucke and Crespi ${ }^{32}$ attempted to predict the best packed structures for snowman-shaped particles and found some of those later predicted for binary hard spheres. While knowledge of the best packed structures can give an indication to the phase behavior of a system at highest densities, it does not give any information on the phase behavior at lower densities nor about the phase boundaries. 
A recent study using molecular dynamics simulations of snowman-shaped particles with various soft potentials ${ }^{33}$ showed that a $\mathrm{NaCl}$ crystal could spontaneously form under compression for diameter ratios below $\sim 0.41$ as one approaches the hard-particle limit. Similarly, for large diameter ratios ( $\gtrsim 0.95$ ) FCC-like structures can form. ${ }^{16,17}$ For intermediate diameter ratios, however, no crystalline structures spontaneously formed, possibly due to the potential crystal structures in this region being kinetically inaccessible. ${ }^{33}$ In this paper, we use computer simulations to map out the phase diagram by evaluating the free energies of candidate crystalline structures which correspond to the close packed structures of binary hard-sphere mixtures. We take the structures found in Ref. 29 to be our candidate crystals, and we place the large and small spheres making up the snowman particles on the lattice sites. As the spheres are tangential there is no difference in the close packed structures of the snowmen and the binary crystals. ${ }^{27}$ Therefore, we define all crystalline structures based on the positions of the constituent spheres of the snowmen.

\section{METHOD}

\section{A. Simulation details}

We perform Monte Carlo (MC) simulations on systems of hard snowman-shaped particles. These particles consist of two hard spheres rigidly bonded at their surfaces (see Fig. 1). We define the shape of these particles by the ratio of the constituent sphere diameters $d=D_{S} / D_{L}$, where $D_{S}$ is the diameter of the smaller sphere and $D_{L}$ is the diameter of the larger sphere. In the limiting case of $d=0$ the snowmen reduce to hard spheres and for $d=1$ they reduce to hard-sphere dimers. We study systems from $d=0$ to $d=1$ in steps of 0.1 , except in the regions of $0.2<d<0.5$ and $0.9<d<1$ where a higher resolution is required to clarify the phase behavior.

To obtain the equations of state (EOS) for a given diameter ratio $d$, we perform constant pressure Monte Carlo (NPT) simulations on a system of $N \sim 500$ snowmen, at temperature $T$ and pressure $P$. We note that only the ratio $P / T$ is relevant in hard-body systems. For all systems studied we perform both compression runs, where we start from an initial isotropic fluid configuration at low pressure and then increase $P$ in small steps, and expansion runs, where we employ one of the candidate crystal structures and decrease the pressure until the crystal melts. We consider the densely packed structures which were predicted for the binary hard-sphere systems in Ref. 29 for the corresponding $d$ values as the candidate crystal structures. We list these in Table I. For each state point (i.e., at each pressure $P$ ) we divide the simulation runs into two parts: an equilibration part of $\sim 2 \times 10^{6} \mathrm{MC}$ cycles, followed by a production part of $\sim 4 \times 10^{6} \mathrm{MC}$ cycles from which we ob-

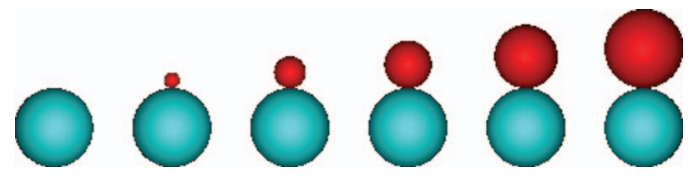

FIG. 1. Snowman particles with $d=D_{S} / D_{L}=0$ to $d=1$, in intervals of 0.2 .
TABLE I. Candidate crystal structures for snowman particles of diameter ratio $d=D_{S} / D_{L}$ ranging from 0.1 to 0.98 , listed from best packed at high pressure to less well packed.

\begin{tabular}{lcccc}
\hline \hline$d=D_{S} / D_{L}$ & \multicolumn{3}{c}{ Candidate structures } \\
\hline 0.1 & $\mathrm{NaCl}$ & $\mathrm{CrB}$ & & \\
0.2 & $\mathrm{NaCl}$ & $\mathrm{CrB}$ & & \\
0.25 & $\mathrm{NaCl}$ & $\mathrm{CrB}$ & & \\
0.3 & $\mathrm{NaCl}$ & $\mathrm{CrB}$ & & \\
0.35 & $\mathrm{NaCl}$ & $\mathrm{CrB}$ & & \\
0.38 & $\mathrm{NaCl}$ & $\mathrm{CrB}$ & & \\
0.4 & $\mathrm{NaCl}$ & $\mathrm{CrB}$ & & \\
0.42 & $\mathrm{NaCl}$ & $\mathrm{CrB}$ & & \\
0.48 & $\mathrm{CrB}$ & $\mathrm{NaCl}$ & & $\gamma \mathrm{CrV}$ \\
0.5 & $\mathrm{CrB}$ & $\mathrm{NaCl}$ & $\gamma \mathrm{CuTi}$ & \\
0.6 & $\mathrm{CrB}$ & $\mathrm{NaCl}$ & $\alpha \mathrm{IrV}$ & $\gamma \mathrm{CuTi}$ \\
0.7 & $\mathrm{CrB}$ & $\alpha \mathrm{IrV}$ & $\mathrm{CsCl}$ & $\mathrm{CrB}$ \\
0.8 & $\gamma \mathrm{CuTi}$ & $\alpha \mathrm{IrV}$ & $\mathrm{CsCl}$ & $\mathrm{CrB}$ \\
0.9 & $\alpha \mathrm{IrV}$ & $\gamma \mathrm{CuTi}$ & $\mathrm{CsCl}$ & \\
0.95 & $\alpha \mathrm{IrV}$ & $F C C^{*}$ & & \\
0.97 & $\alpha \mathrm{IrV}$ & $F C C^{*}$ & & \\
0.98 & $\alpha \mathrm{IrV}$ & $F C C^{*}$ & & \\
\hline \hline
\end{tabular}

tain the density $\rho$ of the system at pressure $P$ and hence the EOS.

\section{B. Crystal structures}

For a large range of $d$ values compression of isotropic phases does not result in the spontaneous formation of crystalline structures. Therefore, in order to obtain the crystalline branches of the EOS we must generate all candidate crystals at high pressure and then expand them until they melt. As the snowmen consist of tangential spheres, we choose to classify their crystalline structures as the structures of the corresponding binary hard-sphere crystals (i.e., the constituent spheres, instead of the centers of mass of the snowmen, are positioned on a crystal lattice). However, in addition to the positions of the constituent spheres, a crystal of snowman particles is also defined by the orientational ordering of the particles, and this gives rise to three types of orientational organization. These are: (i) periodic crystals (PC), in which the constituent spheres have positional order and the snowmen have periodic orientational ordering; (ii) aperiodic crystals (APC), in which the constituent spheres have positional order but there is no repeating orientational ordering of the snowmen, and as such the snowmen centers of mass are positionally aperiodic; (iii) rotator (plastic) crystals (RC), in which the snowmen center of mass positions are on average located on a lattice, but the particles can still rotate (although free rotation is hindered by the surrounding particles). We note that, particularly at high densities, the instantaneous position of a particle can be correlated to its orientation. ${ }^{34}$ For large $d$ values, we also find one additional type of structure in which the constituent spheres lie on a slightly distorted FCC lattice, with the large and small spheres positioned on random sites. As such, this structure has no repeating positional ordering of the constituent spheres, nor repeating orientational or positional ordering of the snowman particles. These structures are 
similar to the 2D aperiodic structures studied in Ref. 35, and also to the aperiodic FCC-like structure found in Ref. 22 to be stable for $d=1$ (tangential dumbbells), and we refer to them as $F C C^{*}$ from hereon in.

For both PCs and RCs, there is only one possible configuration, and to obtain the EOS for a given $d$ only a single set of expansion runs has to be performed. There is, however, a large number of configurations (yet finite for a given number of particles) that we characterize as APCs of a given crystal structure, as there are multiple combinations of snowman orientations with the same corresponding binary crystal structure. The number of possible configurations defines the degeneracy of an APC. For snowman-shaped particles the APC and PC of each crystalline structure are indistinguishable in terms of packing due to the non-penetrating nature of the two constituent spheres. As such, we would expect the PC and different APC configurations of a candidate crystal to have identical EOS, and hence that the periodic crystal structures can be considered as a special case of the APC configurations. We check this by generating and expanding a PC and 3 APC configurations for each candidate crystal. For $F C C^{*}$ structures, there are again multiple configurations, and in this case due to the random positioning of the large and small constituent spheres on the lattice sites, we would expect different configurations to have slightly different EOS, particularly at high densities where packing considerations become important. Because of this, we also generate and expand multiple $F C C^{*}$ configurations for the relevant $d$ values.

We generate the crystal structures using the following method. Firstly, we generate a binary hard-sphere structure of $N_{L}=N_{S} \sim 64$ particles for each of the candidate structures. We then allow these to equilibrate at high pressure, and based on the configuration obtained we produce a larger system of typically $N_{L}=N_{S} \sim 500$ spheres. The exact number varies slightly depending on the crystal structure. Next we connect neighboring spheres of different species to form a periodic crystal structure of snowman-shaped particles. Finally, we use bond-switch moves ${ }^{22}$ to produce APC crystals. For $F C C^{*}$ structures we use the same method, but we start from a crystal with $D_{L}=D_{S}$ from which we form an APC crystal. We then reduce the diameter of one of the constituent spheres (chosen randomly) to give the required $d$ value. This system is finally allowed to equilibrate at high pressure.

Rotator phases form spontaneously during the expansion runs, and in the case of low $d$, during the compression runs. As such, we do not need to generate these configurations.

\section{Free energy calculations}

We determine which crystals are stable and map out the phase diagram using free energy calculations. In order to calculate the Helmholtz free energy $F$ of the isotropic phases we initially use the Widom insertion method ${ }^{36}$ to calculate the chemical potential $\mu$, which is then related to the free energy by

$$
\frac{F}{N}=\mu-\frac{P}{\rho}
$$

To calculate the free energies of the candidate crystal structures we use the thermodynamic integration method to integrate from a reference state. For each crystal of interest the reference state is a non-interacting Einstein crystal with the corresponding structure, for which the free energy can be calculated analytically. ${ }^{37}$ The method we use is similar to that in Ref. 22, which considered hard dumbbells, with some adaptations required to describe snowman-shaped particles.

We begin by considering rotator crystals, which have no fixed orientation but do have positional ordering of the average snowmen centers of mass. We wish to be able to follow a path through phase space that connects an RC to the reference Einstein crystal. To do so, we start by tethering the snowmen with harmonic springs to their corresponding lattice sites in the reference crystal. The coupling potential energy function is given by

$$
\beta U_{k}\left(\mathbf{r}^{N}, \mathbf{u}^{N}, k\right)=k \sum_{i=1}^{N} \frac{\left(\mathbf{r}_{i}-\mathbf{r}_{i, 0}\right)^{2}}{\sigma^{2}},
$$

where $k$ is the spring constant, $\mathbf{r}_{i}-\mathbf{r}_{i, 0}$ is the distance of particle $i$ at position $\mathbf{r}_{i}$ from its corresponding lattice site at $\mathbf{r}_{i, 0}$ and $\mathbf{u}_{i}$ is the orientation of the snowman particle. We take $\sigma=D_{L}$ to be the unit of length in our system. To obtain the lattice site positions $\mathbf{r}_{i, 0}$ of the reference crystal we calculate the average center of mass position of each particle in an equilibrated crystal configuration using constant volume $(N V T)$ simulations at a point of interest. For $k=0$ the particles are completely untethered, and such a system corresponds to the structure under consideration (i.e. the rotator crystal). The spring constant $k$ is then increased gradually to a value $k_{\max }$, at which the particles are completely fixed to their lattice sites. We find that a value $k_{\max } \sim 16000$ is sufficient to fix the particles.

While the particles are now fixed positionally, they can still rotate about their center of mass and hence particle interactions are still possible. To ensure that the particles do not interact as they rotate, we replace the hard-core interaction of the snowmen with a pair potential that allows the softness of the interaction to be tuned. We use the following soft potential:

$\beta U_{\gamma}\left(\mathbf{r}^{N}, \mathbf{u}^{N}, \gamma\right)= \begin{cases}\gamma \sum_{i<j} \sum_{a, b=1}^{2}\left(1-A \frac{\mathbf{r}^{\prime 2}}{\sigma^{2}}\right), & r^{\prime} \leq \sigma_{a b} \\ 0, & r^{\prime}>\sigma_{a b}\end{cases}$

where $\mathbf{r}^{\prime}=\mathbf{r}_{i a}-\mathbf{r}_{j b}$ is the separation of sphere $a=1,2$ of particle $i$ from sphere $b=1,2$ of particle $j, \sigma_{a b}$ is the interaction diameter $\left(\sigma_{11}=D_{1}, \sigma_{22}=D_{2}\right.$, and $\sigma_{12}=\sigma_{21}=\left(D_{1}\right.$ $\left.+D_{2}\right) / 2$ ), and $\gamma$ and $A$ are adjustable parameters. We set $A$ $=0.9$ throughout, ${ }^{22}$ and vary $\gamma$ from 0 to $\gamma_{\max }$. In the limit of $\gamma \rightarrow \infty$, the potential reduces the system to hard snowmen once more, and we find that a value of $\gamma \gtrsim 150$ is sufficient to achieve this behavior. As such, we begin from a maximum value of $\gamma_{\max }=200$, and then slowly reduce $\gamma$ until $\gamma \rightarrow 0$, where the constituent spheres become non-interacting.

We now have the following integration path from the rotator phase to the reference crystal: at $\gamma_{\max }$, where the 
particles behave as hard snowmen, we turn on the spring potential $U_{k}\left(\mathbf{r}^{N}, \mathbf{u}^{N}, k\right)$ by increasing $k$ from 0 to $k_{\max }$ to fix the particles to their lattice sites. At $k_{\max }$ we decrease $\gamma$ to 0 so that the particles cease to interact, and the system reduces to a non-interacting Einstein crystal. The Helmholtz free energy $F$ of a system of $N$ particles at volume $V$ and temperature $T$ is then calculated by integrating over this path, and is given by

$$
\begin{aligned}
\beta F=\beta F_{E} & -\int_{0}^{k_{\max }} d k\left\langle\frac{\partial \beta U_{k}}{\partial k}\right\rangle_{\gamma_{\max }} \\
& +\int_{0}^{\gamma_{\max }} d \gamma\left\langle\frac{\partial \beta U_{\gamma}}{\partial \gamma}\right\rangle_{k_{\max }} \\
=\beta F_{E} & -\int_{0}^{k_{\max }} d k\left\langle\sum_{i=1}^{N} \frac{\left(\mathbf{r}_{i}-\mathbf{r}_{i, 0}\right)^{2}}{\sigma^{2}}\right\rangle \\
& +\int_{0}^{\gamma_{\max }} d \gamma\left\langle\sum_{i<j} \sum_{a, b=1}^{2}\left(1-A \frac{\left(\mathbf{r}_{i a}-\mathbf{r}_{j b}\right)^{2}}{\sigma^{2}}\right)\right\rangle,
\end{aligned}
$$

where $F_{E}$ is the Helmholtz free energy of a non-interacting Einstein crystal together with the center of mass correction. In our case this is given by

$$
\begin{aligned}
\beta F_{E}= & -\frac{3(N-1)}{2} \ln \left(\frac{\pi}{k_{\max }}\right)+\ln \left(\frac{\rho \sigma^{3}}{N^{3 / 2}}\right) \\
& +N \ln \left(\Lambda_{r}^{2}\right)+N \ln \left(\frac{\Lambda_{t}^{3}}{\sigma^{3}}\right) .
\end{aligned}
$$

$\Lambda_{r}$ is given by

$$
\Lambda_{r}=\sqrt{\frac{\beta h^{2}}{8 \pi^{2} I}}
$$

where $h$ is the Planck constant and $I$ is the moment of inertia of the particle. $\Lambda_{t}$ is the de Broglie wavelength given by

$$
\Lambda_{t}=\sqrt{\frac{\beta h^{2}}{2 \pi m}}
$$

where $m$ is the particle mass. We calculate the integrands in Eq. (4) by performing NVT Monte Carlo simulations and using these together with Eq. (5) we calculate the free energy of all rotator phases.

We now consider PC, APC, and $F C C^{*}$ structures as defined in Sec. II B. In order to obtain the free energies we follow the same procedure as outlined above for RCs. However, we also add an additional term to the potential in Eq. (2), which couples the particle orientations to the corresponding orientations in the reference crystal. We use $N V T$ simulations to calculate the average positions $\mathbf{r}_{i, 0}$ and orientations $\mathbf{u}_{i, 0}$ of each particle within the crystal. Eq. (2) then becomes

$$
\beta U_{k}\left(\mathbf{r}^{N}, \mathbf{u}^{N}, k\right)=k \sum_{i=1}^{N}\left(\frac{\left(\mathbf{r}_{i}-\mathbf{r}_{i, 0}\right)^{2}}{\sigma^{2}}+\frac{1-\cos \theta_{i}}{2}\right),
$$

where $\theta_{i}$ is the angle between the orientations of particle $i$ and the corresponding particle in the reference crystal, and is given by $\cos ^{-1}\left(\mathbf{u}_{i} \cdot \mathbf{u}_{i, 0}\right)$. Equation (4) is therefore modified to become

$$
\begin{aligned}
\beta F=\beta F_{E} & -\int_{0}^{k_{\max }} d k\left\langle\frac{\partial \beta U_{k}}{\partial k}\right\rangle_{\gamma_{\max }} \\
& +\int_{0}^{\gamma_{\max }} d \gamma\left\langle\frac{\partial \beta U_{\gamma}}{\partial \gamma}\right\rangle_{k_{\max }}-\ln \Omega \\
=\beta F_{E} & -\int_{0}^{k_{\max }} d k\left\langle\sum_{i=1}^{N}\left(\frac{\left(\mathbf{r}_{i}-\mathbf{r}_{i, 0}\right)^{2}}{\sigma^{2}}+\frac{1-\cos \theta_{i}}{2}\right)\right\rangle \\
& +\int_{0}^{\gamma_{\max }} d \gamma\left\langle\sum_{i<j} \sum_{a, b=1}^{2}\left(1-A \frac{\left(\mathbf{r}_{i a}-\mathbf{r}_{j b}\right)^{2}}{\sigma^{2}}\right)\right\rangle \\
& -\ln \Omega,
\end{aligned}
$$

where we again integrate over both the spring and soft potentials to relate the free energy of each crystal structure to that of the corresponding reference crystal. Note that there is now an additional term $\ln \Omega$, where $\Omega$ is the degeneracy of the crystalline phase (i.e., the number of possible configurations of a structure). We calculate the value of $\Omega$ using the series expansion method given in Ref. 38, which has been shown to be accurate for sufficiently large systems. ${ }^{22}$ The zeroth order term $\Omega_{0}$ of this expansion, which reduces to the Bethe approximation, depends solely on the number of smaller spheres that touch each larger sphere, which we denote as $q$, and is given by

$$
\Omega_{0}=q^{N}\left(1-\frac{1}{q}\right)^{N(q-1)}
$$

The higher order terms vary even for structures with the same $q$ value, and we calculate these up to 8th order and show the calculated values of $\ln \Omega$ in Table II. Typically we find that the difference between the values obtained from the high order expansion and from the zeroth order term is of the order of a few percent. For $F C C^{*}$ crystalline phases, $\Omega$ also takes into account the positional degeneracy, which can be calculated by counting the number of possible particle configurations and correcting for periodic boundary conditions. Finally, the free energy of the non-interacting Einstein crystal $F_{E}$ with

TABLE II. Degeneracy term per particle $\ln \Omega / N$ of candidate crystal structures for snowman particles. $q$ gives the number of smaller spheres that touch each large sphere.

\begin{tabular}{lcc}
\hline \hline Crystal structure & $q$ & $\ln \Omega / N$ \\
\hline $\mathrm{NaCl}$ & 6 & 0.8945 \\
$\mathrm{CrB}$ & 6 & 0.8933 \\
$\mathrm{CrB}$ & 7 & 1.0279 \\
$\alpha \mathrm{IrV}$ & 6 & 0.8864 \\
$\alpha \mathrm{IrV}$ & 8 & 1.1504 \\
$\gamma \mathrm{CuTi}$ & 5 & 0.7293 \\
$\gamma \mathrm{CuTi}$ & 4 & 0.5423 \\
$F C C^{*}$ & 12 & 2.2114 \\
\hline
\end{tabular}


the center of mass correction becomes

$$
\begin{aligned}
\beta F_{E}= & -\frac{3(N-1)}{2} \ln \left(\frac{\pi}{k_{\max }}\right)+\ln \left(\frac{\rho \sigma^{3}}{N^{3 / 2}}\right) \\
& +N \ln \left(\Lambda_{r}^{2}\right)+N \ln \left(\frac{\Lambda_{t}^{3}}{\sigma^{3}}\right)-N \ln \left(J\left(k_{\text {max }}\right)\right),
\end{aligned}
$$

where the final term arises due to the orientational part of Eq. (8), and is given by

$$
J(k)=\int_{-1}^{1} e^{k(x-1) / 2} d x=\frac{2\left(1-e^{-k}\right)}{k} .
$$

In order to account for finite size effects, for all crystalline phases we perform free energy calculations for various $N$ values, and extrapolate the results to $N \rightarrow \infty$.

Using the free energies and equation of state calculations, we determine the coexistence regions by equating both the pressure and chemical potential in phase $i$ at density $\rho_{i}$ and in phase $j$ at density $\rho_{j}$

$$
\begin{aligned}
& P\left(\rho_{i}\right)=P\left(\rho_{j}\right) \\
& \mu\left(\rho_{i}\right)=\mu\left(\rho_{j}\right) .
\end{aligned}
$$

Finally, we use these to construct the phase diagram.

\section{RESULTS}

\section{A. Crystal structures}

We first comment on the candidate crystal structures we constructed using the method described in Sec. II B. As starting configurations we employ one of the dense packed crystal structures as predicted for the equimolar binary hard-sphere mixtures in Ref. 29. We refer the interested reader to the supplementary information of Ref. 27 for data necessary to construct the close packed structures. We then perform NPT Monte Carlo simulations for the snowman particles at very high pressures. Figure 2 shows example snapshots of each of the candidate crystalline structures considered. We note that, with the exception of the $\mathrm{CsCl}$ structure, all of the candidates considered are for some range of $d$ values the best packed structure. We also find that for some $d$ values the generated candidate structures can reconfigure into other structures (such as $\mathrm{CsCl}$ structures forming $\alpha \mathrm{IrV}$ structures, $\gamma \mathrm{CuTi}$ structures forming $\mathrm{CrB}$ structures, etc.), which allows us to discard some of the potential candidate structures in advance of performing the free energy calculations. We do note, however, that we observe no transitions between the various APC configurations of a candidate structure, or between the various $F C C^{*}$ structures, during our simulation runs.

In most cases the highest packings that we find agree with those given in Refs. 27 and 29. However, upon further compression we find in several cases that systems with certain structures can maximize their packing by regularly distorting the initial lattice configurations. For $d>0.414$, we find that a system with the standard $\mathrm{NaCl}$ structure can significantly increase its packing by separating alternating pairs of large spheres that touch at close packing to fit better the smaller spheres in between (see Fig. 3). Similarly, for $0.75 \lesssim d$
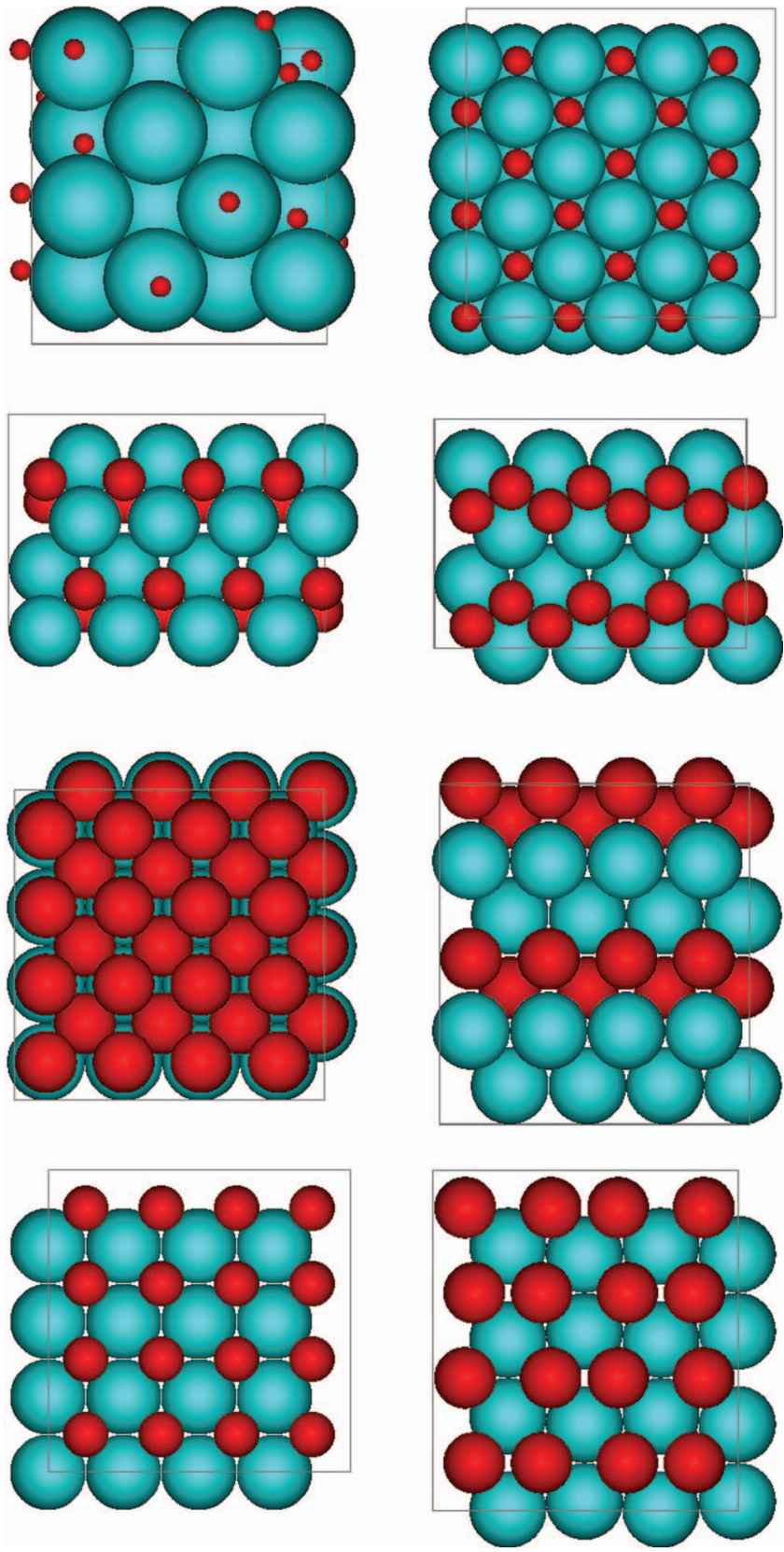

FIG. 2. Examples of candidate crystal structures for snowman particles (see text). Light blue spheres are larger spheres with diameter $D_{L}$, red spheres are smaller spheres with diameter $D_{S}$. Top row, left: rotator crystal (RC) with diameter ratio $d=D_{S} / D_{L}=0.2$. Top row, right: $\mathrm{NaCl}$ with $d=0.4$. Second row: two planes of $\mathrm{CrB}$ with $d=0.5$. Third row: two planes of $\gamma \mathrm{CuTi}$ with $d$ $=0.8$. Bottom row, left: $\mathrm{CsCl}$ with $d=0.6$. Bottom row, right: $\alpha \mathrm{IrV}$ with $d$ $=0.8$.

$\lesssim 0.95$, the $\alpha \mathrm{IrV}$ structure changes from having the same number of small spheres touching each large sphere at intermediate pressures, to alternating numbers of contacts at high pressures (i.e., half of the large spheres have 4 small sphere neighbors and half have 8, see Fig. 3). When we expand these modified structures, we find that they change continuously into the initial, simpler, crystalline structures, at a density at which the particles have sufficient free space that the distortion is no longer necessary.

In Figure 4 we show the best packed densities for all the candidate crystals considered. As noted previously, in some 

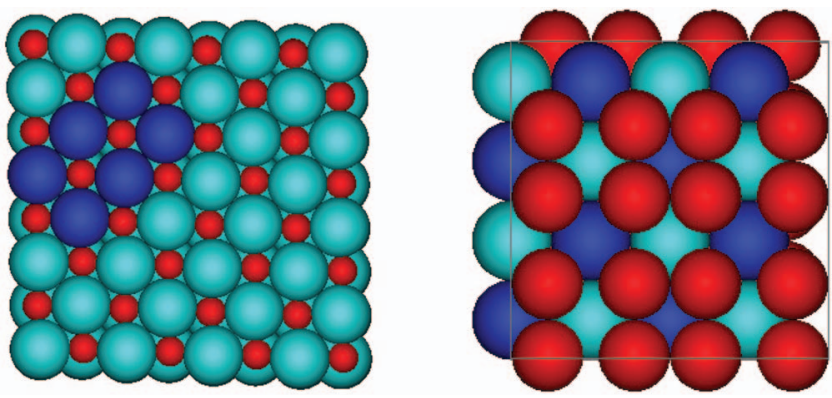

FIG. 3. Examples of modified candidate crystal structures for snowman particles (see text). Light blue spheres are larger spheres with diameter $D_{L}$, red spheres are smaller spheres with diameter $D_{S}$. Left: $\mathrm{NaCl}$ with diameter ratio $d=0.45$. The dark blue region highlights the modified behavior where we see that pairs of larger spheres alternate between touching and being separated. Right: $\alpha \operatorname{IrV}$ with $d=0.85$, where dark blue spheres are larger spheres with 4 neighbors and light blue spheres are larger spheres with 8 neighbors.

cases the candidate structures may have either formed modified structures or changed into other structures. However, we find that the crystal structures only change into other crystal structures for $d$ values where the candidate crystal under consideration is anyway not the best packed structure. We finally note that the number of contacts that each sphere has with spheres of different diameter at highest packing determines the number of ways we can connect the spheres into snowman particles, and hence also the degeneracy of the APC structures.

\section{B. Equations of state}

We now examine the equations of state (EOS) calculated from our simulation runs, beginning with the compressions of the isotropic fluid phases. For systems with $d \lesssim 0.3$ we see the spontaneous formation of rotator phases upon compression (see Fig. 5(a) for the EOS for $d=0.1$ ), with the center of mass of the particles located on average on an FCC lattice. In the case of $d \lesssim 0.2$, further compression of the obtained RCs

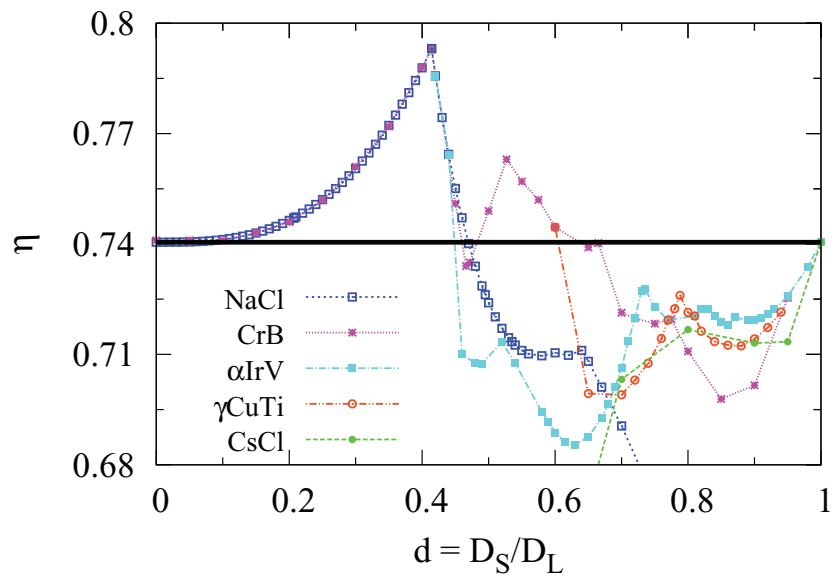

FIG. 4. Close packing densities (in packing fraction $\eta=N v_{0} / V$ with $v_{0}$ the particle volume) of candidate crystal structures for snowman-shaped particles as a function of diameter ratio $d$, constructed using the method outlined in Sec. II B. The legend indicates the crystal structures that the compression runs start from and in some cases the structures have rearranged from this to form another structure.
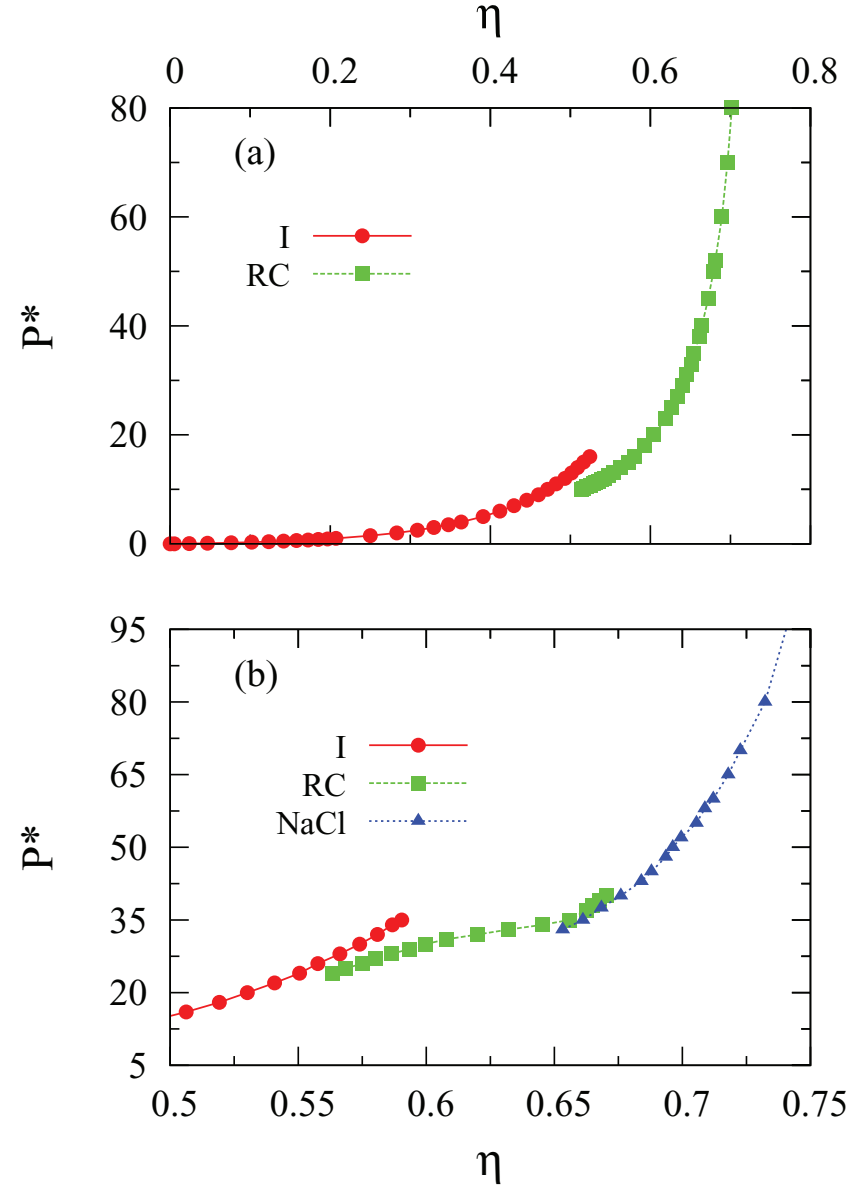

FIG. 5. Equations of state of (a) isotropic and rotator phases for $d=0.1$ and (b) isotropic, rotator and $\mathrm{NaCl}$ phases for $d=0.4 . \eta=N v_{0} / V$ is the packing fraction with $v_{0}$ the particle volume, and $P^{*}=\beta P \sigma^{3}$ is the reduced pressure. I denotes the isotropic phase.

does not result in any further phase transitions. However, at higher packings we observe that the large spheres, rather than the particle centers of mass, are positioned on an FCC lattice, while the smaller spheres can still move within the free space. We note that in this case the gaps left by the larger spheres are large enough to fit multiple smaller spheres. For $0.2 \lesssim d$ $\lesssim 0.3$, where the gaps are no longer large enough, compression of the RCs results in the formation of an aperiodic structure resembling the $\mathrm{NaCl}$ crystal.

For $d \gtrsim 0.3$ compression of the isotropic fluid phase does not result in any phase transitions and therefore we must expand one of the generated candidate crystal structures. For each crystal structure we have examined at least 3 different aperiodic structures as well as the periodic structure. We find that each of these have the same EOS, and as an example we show this for the CrB crystal with $d=0.7$ in Fig. 6(a). As mentioned previously, this is to be expected since the constituent spheres of the snowman particles are joined at their surfaces and hence arranging the snowman orientations periodically would not result in better overall packing. As before, we conclude that the PCs for snowman particles can be considered as a special case of the corresponding APC crystalline structures. 

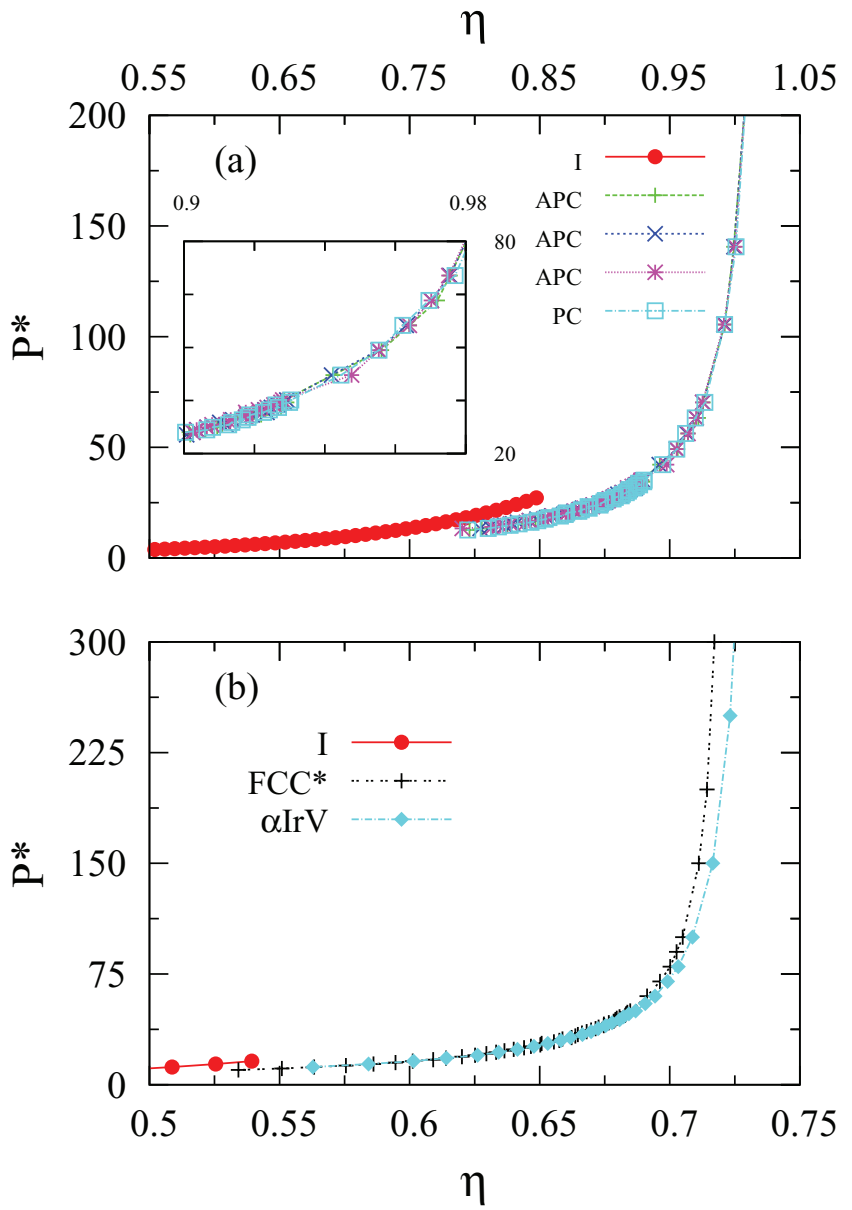

FIG. 6. Equations of state of (a) isotropic, three aperiodic and periodic $\mathrm{CrB}$ phases for $d=0.7$ (inset shows a smaller range of packing fractions) and (b) isotropic, $F C C^{*}$ and $\alpha \operatorname{IrV}$ phases for $d=0.98 . \eta=N v_{0} / V$ is the packing fraction with $v_{0}$ the particle volume, and $P^{*}=\beta P \sigma^{3}$ is the reduced pressure. I denotes the isotropic phase.

For systems upto $d \sim 0.48$ we find that upon expansion the candidate crystals transition into RC phases, where once again the particle centers of mass are on average positioned on an FCC lattice. Once formed, we recompress the RCs, and we find that for $0.2 \lesssim d \lesssim 0.48$ this does not result in the reformation of the candidate structure, but instead results in the continuous change to a structure with fixed particle orientations, resembling the $\mathrm{NaCl}$ crystal. As an illustration of this behavior in Fig. 5(b) we show the EOS for $d=0.4$. Further expansion of all RCs results in a transition to an isotropic phase.

For $d \gtrsim 0.48$ we observe direct APC-isotropic phase transitions upon expansion of the crystal phases. For $d \gtrsim 0.97$ we find that the EOS of $\alpha \operatorname{Ir} V$ (which is the crystal structure with highest packing in this $d$ range) and the $F C C^{*}$ crystals are the same at low pressure (see Fig. 6(b)), while they differ significantly at higher pressures. This indicates that for these small anisotropies in the sphere diameters, the spheres only become distinguishable at high densities.

We finally note that in some cases we observe crystalcrystal transformations, explained in Sec. III A. For the $\mathrm{NaCl}$ structures (with $d \gtrsim 0.414$ ), we see that the modified structure described in the Sec. III A converts into the standard $\mathrm{NaCl}$ structure upon expansion, with this transformation having no obvious effect on the EOS. Upon recompression we observe the same continuous change from the standard to the modified structure. For the modified $\alpha \mathrm{IrV}$ structure (found for $0.75 \lesssim d$ $\lesssim 0.95$ ) the process is identical, with a reversible continuous transformation from modified to standard structure observed.

\section{Phase diagram}

We now use the methods described in Sec. II C to calculate the free energies of the isotropic fluid phases and the candidate crystal structures. We determine the stable phases at each $d$ value and calculate the coexistence densities at all phase transitions to construct the phase diagram of snowmanshaped particles. The predicted phase diagram is shown in Fig. 7. We note that, as stated previously, for all non-RC structures studied we find that the stable structures are all orientationally aperiodic. Indeed, we find that the free energies of the PC structures are identical to that of the corresponding APC structures except for the degeneracy term $\ln \Omega$ (see Eq. (9)). As such, from hereon all crystalline phases discussed are aperiodic unless stated otherwise.

We predict the existence of stable isotropic fluid and rotator phases for $d \lesssim 0.45$, although the RCs formed during the expansion of candidate crystals for a slightly larger range of $d$ values ( $d \lesssim 0.48$ ). For comparison, in systems of hard dumbbells (with diameter ratio $d=1$ ) a rotator phase is stable for sphere separations of $\lesssim 0.38 .{ }^{22}$ A dumbbell particle therefore has a slightly shorter length of the major axis at the rotator phase triple point than a snowman particle does at the corresponding rotator phase triple point, but has a larger particle volume $(\sim 1.4$ times as large). We note that the RC phase initially becomes slightly more stable with respect to the isotropic phase as $d$ goes from 0 to 0.1 , before becoming increasingly less stable at larger $d$ values.

In the region where $d \lesssim 0.2$ we find no further phase transitions (as discussed in Sec. III B). For $0.2 \lesssim d \lesssim 0.4$ we predict that with increasing density the RC transitions into an $\mathrm{NaCl}$ crystal structure. The range of $d$ values where $\mathrm{NaCl}$ phases are stable is roughly the same as the range of size ratios for which $\mathrm{NaCl}$ phases were observed to form spontaneously in Ref. 33. We also see that as $d$ increases the $\mathrm{NaCl}$ phases become increasingly more stable with respect to the $\mathrm{RC}$ phases. Clearly, as $d$ increases the free energy gain associated with freely rotating particles is diminished as particles interact more strongly during rotations, and hence an orientationally ordered phase is favored.

The behavior in the region $0.4 \lesssim d \lesssim 0.5$ is more complex. At $d=0.42$ we find both isotropic-RC and $\mathrm{RC}-\mathrm{NaCl}$ phase transitions, while at $d=0.48$ we only find an isotropic$\mathrm{CrB}$ phase transition. This leads us to believe that there is a point where the rotator and $\mathrm{NaCl}$ phases stop being stable and are replaced by the $\mathrm{CrB}$ crystalline phase. By extrapolating our data, we estimate that this point is located at $d \sim 0.46$, which is also close to the point where the $\mathrm{CrB}$ structure begins to have better packing than the $\mathrm{NaCl}$ structure (at $d \sim 0.47$, see Fig. 4).

From $d \sim 0.47$ to $d \sim 0.6$ we find only isotropic-CrB coexistence. At $d=0.7$ we find that the $\alpha \operatorname{IrV}$ phase becomes 
stable at intermediate densities while the $\mathrm{CrB}$ phase is still stable at higher densities, i.e., the phase behavior changes from an isotropic-CrB phase transition to isotropic- $\alpha \operatorname{IrV}$ and $\alpha \mathrm{IrV}-\mathrm{CrB}$ phase transitions. We expect there to be a point where $\alpha \mathrm{IrV}$ emerges as the stable phase at intermediate densities, and by extrapolating our results we predict this to be at $d \sim 0.68$. The $\mathrm{CrB}$ phase, however, is the best packed structure for $0.47 \lesssim d \lesssim 0.72$, and we thus expect to find an $\alpha \mathrm{IrV}$ $\mathrm{CrB}$ phase transition for $0.68 \lesssim d \lesssim 0.72$.

The stability of the $\alpha \operatorname{IrV}$ phase in this region can be understood if we consider the effect of degeneracy on the free energy. In Fig. 8 we show both the EOS and the free energy $F$ with the degeneracy term $\ln \Omega$ added (see Eq. (9)), of both the $\alpha \mathrm{IrV}$ and $\mathrm{CrB}$ crystalline phases for the system with $d=0.7$. This allows us to compare the non-degeneracy related contributions to the free energy. As can be seen, the EOS of both systems lie on top of each other at intermediate densities, as do the $F+\ln \Omega$ lines (up to $\eta \sim 0.65$ ). However, in the $\alpha \operatorname{IrV}$ phase there is a larger number of touching large-small spheres $(q=8)$ than in the CrB phase $(q=6)$, which results in a larger number of possible APC structures and therefore a larger $\ln \Omega$ term. This results in a lower total free energy, and with it the stability of the $\alpha \operatorname{IrV}$ phase. Clearly, the orientational degrees of freedom of the snowman particles are responsible for stabilizing the $\alpha \mathrm{IrV}$ phase with respect to the $\mathrm{CrB}$ phase. This presents an intriguing scenario where we can potentially stabilize binary crystalline phases with respect to other, better packed, phases by fusing the spheres to form snowman particles.

For $d$ values larger than $\sim 0.72$ the $\mathrm{CrB}$ phase is no longer the best packed structure and we find the $\alpha \operatorname{IrV}$ phase to be the best packed structure up to $d \sim 0.78$. For $0.78 \lesssim d \lesssim 0.81$ we see from Fig. 4 that $\gamma \mathrm{CuTi}$ has the best packing, and indeed, at $d=0.8$, based on our free energy calculations, we find this to be the stable structure at high densities. However, at intermediate densities we find that the $\alpha \operatorname{IrV}$ phase is still stable, and at lower densities we observe isotropic- $\alpha \mathrm{IrV}$ coexistence.

For $d \gtrsim 0.81$ we find that $\alpha \operatorname{IrV}$ is again the best packed structure and at high densities we find this phase to be stable all the way up to $d=1$. At $d=0.9$ we observe only isotropic- $\alpha \mathrm{IrV}$ coexistence, but for $0.9 \lesssim d \lesssim 1$ we also expect a stable $F C C^{*}$ phase to emerge at intermediate densities. To check this, we perform free energy calculations for snowman particles with $d=0.95,0.97$ and 0.98 , finding isotropic$F C C^{*}$ coexistence at $d=0.97$ and 0.98 , but not at $d=0.95$. This closely matches the results of Ref. 33 . We note that the $F C C^{*}$ phase has a similar EOS at low densities to the $\alpha \operatorname{IrV}$ phase (Fig. 6(b)), but has the advantage of a higher degree of degeneracy due to the positional aperiodicity of the constituent spheres, as well as the orientational aperiodicity of the composite particle. It is this higher degeneracy that stabilizes the $F C C^{*}$ phase. As the density is increased, we see from Fig. 6(b) that the $\alpha \operatorname{IrV}$ phase becomes better packed than the $F C C^{*}$ phase, and becomes stable. At $d=1$, the two phases are identical and are also identical to the aperiodic crystal phase found for tangential hard dumbbells in Ref. 22.

Comparing the structures we have found to be stable for snowman particles with those found for binary hard-sphere mixtures ${ }^{39-45}$ we note that only the $\mathrm{NaCl}$ crystal structure is predicted to be stable for binary hard-sphere mixtures with size ratios $d=0.3,{ }^{45} d=0.414$, and $0.45,{ }^{40}$ while the $\mathrm{CrB}$, $\alpha \mathrm{IrV}$ and $\gamma \mathrm{CuTi}$ structures are never found to be stable. The supplementary information of Ref. 44 lists all the binary crystal structures which are predicted to be stable in the phase diagrams of binary hard-sphere mixtures obtained from full free energy calculations for various size ratios.

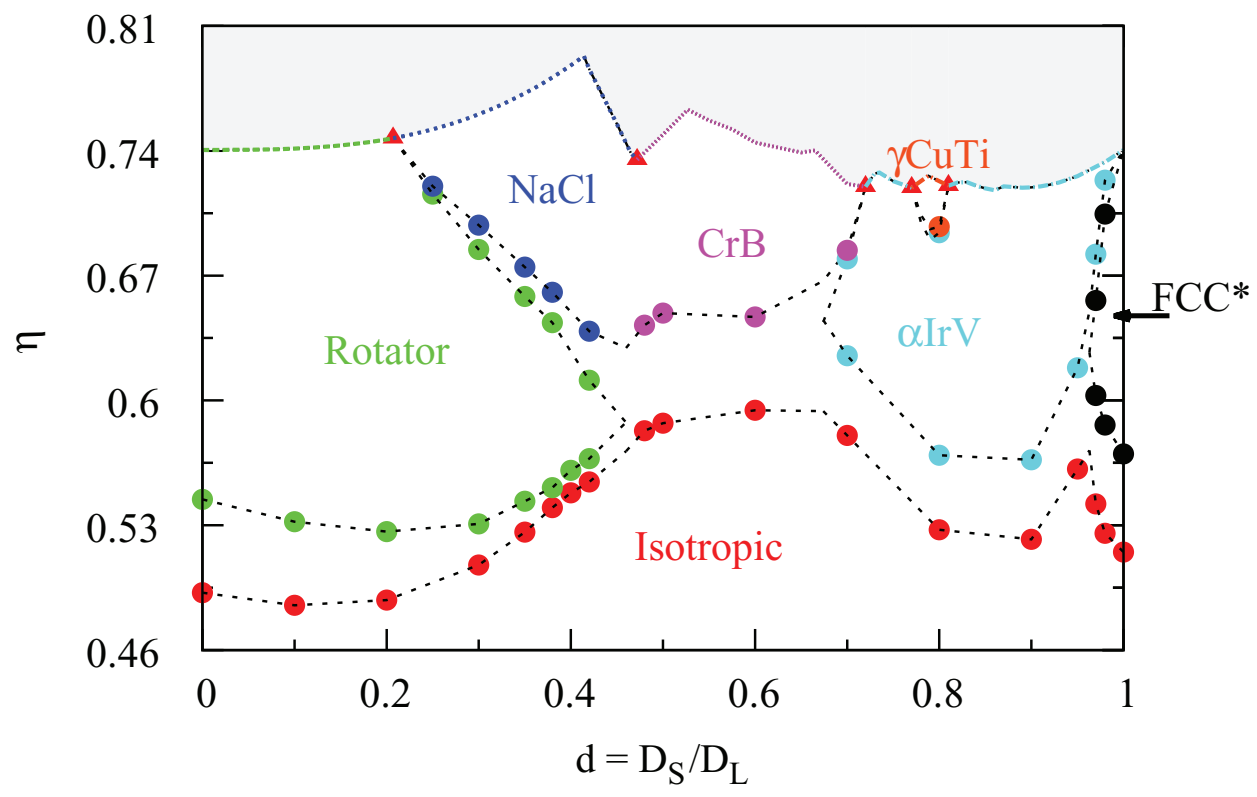

FIG. 7. Phase diagram of snowman particles in the $d-\eta$ representation, with $d=D_{S} / D_{L}$ ranging from 0 (the hard sphere) to $d=1$ (the tangential dumbbell) and $\eta=N v_{0} / V$ (where $v_{0}$ is the snowman volume for a given $d$ value). Circles indicate coexisting phases, while the lines are intended to guide the eye. At the top of the plot we indicate the density of closest packing, with triangles indicating the crossover from one close packed structure to another. Coexistence densities for $d=0$ are taken from Ref. 10 and for $d=1$ they are taken from Ref. 22. 

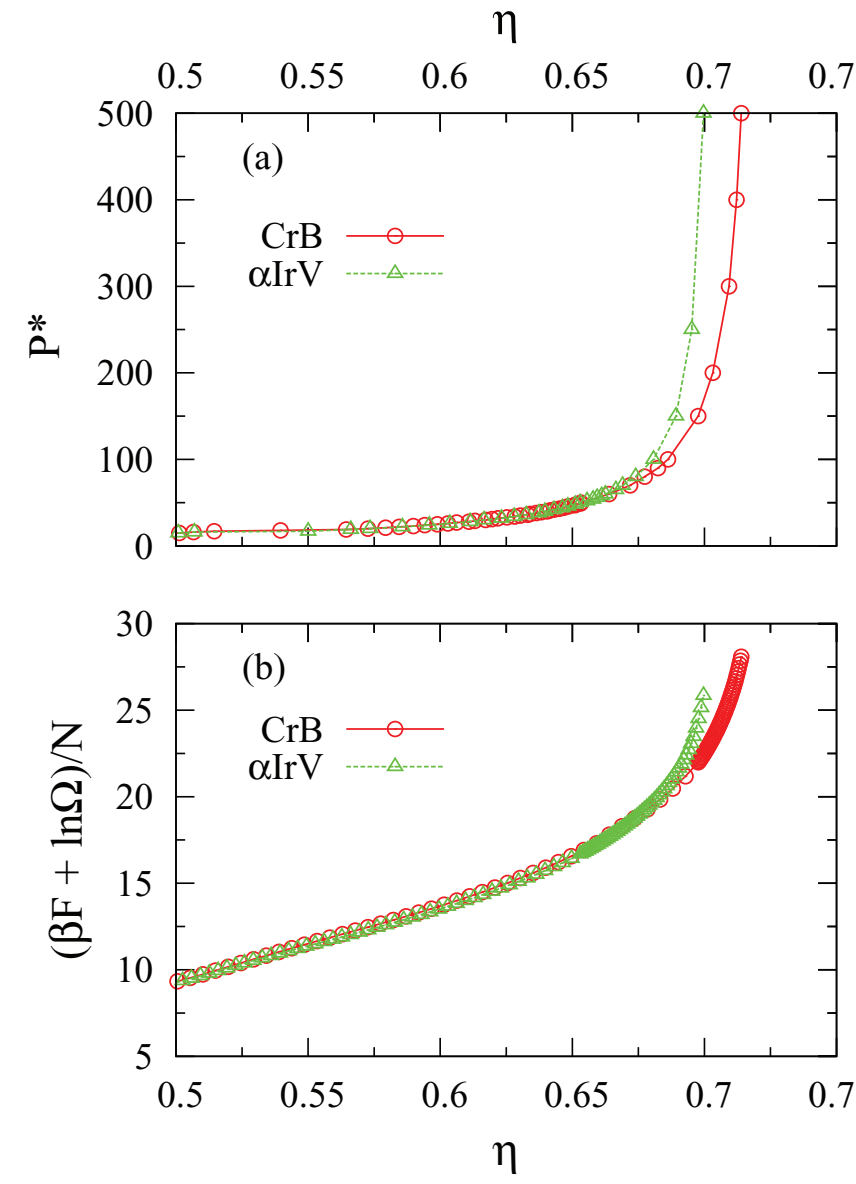

FIG. 8. Data for $\mathrm{CrB}$ (red circles) and $\alpha \operatorname{IrV}$ (green triangles) phases of snowman-shaped particles with diameter ratio $d=0.7$. (a) Equations of state where $\eta=N v_{0} / V$ is the packing fraction with $v_{0}$ the particle volume, and $P^{*}=\beta P \sigma^{3}$ is the reduced pressure. (b) Free energy per particle $F / N$ with degeneracy term per particle $\ln \Omega / N$ added (see Eq. (9)). For the $\mathrm{CrB}$ phase $\ln \Omega / N=0.8933$, while for the $\alpha \operatorname{Ir} V$ phase $\ln \Omega / N=1.1504$.

\section{CONCLUSIONS}

Using Monte Carlo simulations and calculating free energies we have determined the phase diagram of hard snowmanshaped particles. We find the phase behavior of these systems to be extremely rich, with isotropic, rotator crystal, the orientationally aperiodic snowman equivalents of the binary $\mathrm{NaCl}$, $\mathrm{CrB}, \alpha \mathrm{IrV}$ and $\gamma \mathrm{CuTi}$ crystal structures and the snowmanspecific $F C C^{*}$ phases.

At low diameter ratios $d$ we find along with the isotropic phase rotator phases. Increasing the diameter ratio suppresses the rotator phase and orientational ordering becomes favored. For intermediate $d$ values we find the above mentioned crystal phases, all of which are orientationally aperiodic. For large $d$ values (as $d$ approaches 1 ) we find a region where $F C C^{*}$ is stable. We note that all the stable crystalline phases found are orientationally aperiodic as the specific ordering of particles does not affect the packing but lowers the free energy due to a higher degeneracy.

At very high densities we find that the best packed structure is always the stable phase, although in some cases the snowman equivalents of the standard binary crystal structures become modified in order to give better packing. However, at intermediate densities we find that for some $d$ values additional crystalline phases can be stabilized. In such cases we observe that two crystal structures have similar equations of state at intermediate densities, and that the one with the higher degeneracy emerges as the stable phase.

In conclusion, we have shown that the phase diagram of snowman shaped particles is very rich and offers the possibility of forming crystalline phases analogous to and beyond those predicted for binary hard-sphere mixtures. We hope that our research inspires further experimental and theoretical study on these particles.

\section{ACKNOWLEDGMENTS}

This work is financed by a NWO-VICI grant and is part of the research program of the Stichting door Fundamenteel Onderzoek der Materie (FOM) which is financially supported by the Nederlandse organisatie voor Wetenschappelijk Onderzoek (NWO).

${ }^{1}$ S. C. Glotzer and M. J. Solomon, Nature Mater. 6, 557 (2007).

${ }^{2}$ A. van Blaaderen, Science 301, 470 (2003).

${ }^{3}$ V. N. Manoharan, M. T. Elsesser, and D. J. Pine, Science 301, 483 (2003).

${ }^{4}$ M. Okubo, Z. Wang, E. Ise, and H. Minami, Colloid Polym. Sci. 279, 976 (2001).

${ }^{5}$ S. Reculusa, C. Poncet-Legrand, A. Perro, E. Duguet, E. Bourgeat-Lami, C. Mingotaud, and S. Ravaine, Chem. Mater. 17, 3338 (2005).

${ }^{6}$ A. Perro, S. Reculusa, E. Bourgeat-Lami, E. Duguet, and S. Ravaine, Colloids Surf., A 284, 78 (2006).

${ }^{7}$ D. Nagao, M. Hashimoto, K. Hayasaka, and M. Konno, Macromol. Rapid Commun. 29, 1484 (2008).

${ }^{8}$ D. J. Kraft, J. Groenewold, and W. K. Kegel, Soft Matter 5, 3823 (2009).

${ }^{9}$ D. Nagao, C. M. van Kats, K. Hayasaka, M. Sugimoto, M. Konno, A. Imhof, and A. van Blaaderen, Langmuir 26, 5208 (2010).

${ }^{10}$ W. G. Hoover and F. H. Ree, J. Chem. Phys. 49, 3609 (1968).

${ }^{11}$ B. J. Alder, W. G. Hoover, and D. A. Young, J. Chem. Phys. 49, 3688 (1968).

${ }^{12}$ L. V. Woodcock, Nature (London) 385, 141 (1997).

${ }^{13}$ P. G. Bolhuis, D. Frenkel, S. C. Mau, and D. A. Huse, Nature (London) 388, 236 (1997).

${ }^{14}$ S. Mau and D. A. Huse, Phys. Rev. E 59, 4396 (1999).

${ }^{15}$ K. Wojciechowski, Phys. Lett. A 122, 377 (1987).

${ }^{16}$ J. D. McCoy, S. Singer, and D. Chandler, J. Chem. Phys. 87, 4853 (1988).

${ }^{17}$ S. J. Smithline, S. W. Rick, and A. D. J. Haymet, J. Chem. Phys. 88, 2004 (1988).

${ }^{18}$ K. W. Wojciechowski, D. Frenkel, and A. C. Branka, Phys. Rev. Lett. 66, 3168 (1991).

${ }^{19}$ C. Vega, E. P. A. Paras, and P. A. Monson, J. Chem. Phys. 96, 9060 (1992).

${ }^{20}$ C. Vega, E. P. A. Paras, and P. A. Monson, J. Chem. Phys. 97, 8543 (1992).

${ }^{21}$ M. Kowalik and K. W. Wojciechowski, J. Non-Cryst. Solids 354, 4354 (2008).

${ }^{22}$ M. Marechal and M. Dijkstra, Phys. Rev. E 77, 061405 (2008).

${ }^{23}$ P. Bartlett, R. H. Ottewill, and P. N. Pusey, J. Chem. Phys. 93, 1299 (1990).

${ }^{24}$ P. Bartlett, R. H. Ottewill, and P. N. Pusey, Phys. Rev. Lett. 68, 3801 (1992).

${ }^{25}$ N. Hunt, R. Jardine, and P. Bartlett, Phys. Rev. E 62, 900 (2000).

${ }^{26}$ M. J. Murray and J. V. Sanders, Philos. Mag. A 42, 721 (1980).

${ }^{27}$ J. K. Kummerfeld, T. S. Hudson, and P. Harrowell, J. Phys. Chem. B 112, 10773 (2008).

${ }^{28}$ T. S. Hudson and P. Harrowell, J. Phys.: Condens. Matter 23, 194103 (2011).

${ }^{29}$ L. Filion and M. Dijkstra, Phys. Rev. E 79, 046714 (2009).

${ }^{30}$ L. Filion, M. Marechal, B. van Oorschot, D. Pelt, F. Smallenburg, and M. Dijkstra, Phys. Rev. Lett. 103, 188302 (2009).

${ }^{31}$ A. P. Hynninen, L. Filion, and M. Dijkstra, J. Chem. Phys. 131, 046714 (2009).

${ }^{32}$ D. P. Stucke and V. H. Crespi, Nano Lett. 3, 1183 (2003).

${ }^{33}$ A. Šarić, B. Bozorgui, and A. Cacciuto, J. Phys. Chem. B 115, 7182-7189 (2011). 
${ }^{34}$ A. Brańka and K. W. Wojciechowski, Mol. Phys. 78, 1513 (1993).

${ }^{35}$ K. W. Wojciechowski, Phys. Rev. B 46, 26 (1992).

${ }^{36}$ E. Widom, J. Chem. Phys. 39, 2808 (1963).

${ }^{37}$ D. Frenkel and B. Smit, Understanding Molecular Simulation, 2nd ed. (Academic, 2001) .

${ }^{38}$ J. F. Nagle, Phys. Rev. 152, 190 (1966).

${ }^{39}$ M. Dijkstra, R. van Roij, and R. Evans, Phys. Rev. E 59, 5744 (1999).

${ }^{40}$ E. Trizac, M. D. Eldridge, and P. A. Madden, Mol. Phys. 90, 675 (1997).
${ }^{41}$ M. D. Eldridge, P. A. Madden, P. N. Pusey, and P. Bartlett, Mol. Phys. 84, 395 (1995).

${ }^{42}$ M. D. Eldridge, P. A. Madden, and D. Frenkel, Nature 365, 34 (1993).

${ }^{43}$ W. Kranendonk and D. Frenkel, Mol. Phys. 72, 679 (1991).

${ }^{44}$ W. H. Evers, B. de Nijs, F. Filion, S. Castillo, M. Dijkstra, and D. Vanmaekelbergh, Nano Lett. 10, 4235 (2010).

${ }^{45}$ L. Filion, M. Hermes, R. Ni, E. C. M. Vermolen, A. Kuijk, C. G. Christova, J. C. P. Stiefelhagen, T. Vissers, A. van Blaaderen, and M. Dijkstra, Phys. Rev. Lett. 107, 168302 (2011). 